\title{
LA EDUCACIÓN EN EL CORAZÓN DE LAS HUMANIDADES ${ }^{1}$
}

\section{Education at the Heart of the Humanities}

\author{
Chris HIGGINS \\ Boston College. EE. UU. \\ christopher.higgins@bc.edu \\ https://orcid.org/0000-0002-2443-0369
}

Fecha de recepción: 03/03/2021

Fecha de aceptación: 01/08/2021

Fecha de publicación en línea: 01/01/2022

Cómo citar este artículo: Higgins, Ch. (2022). La educación en el corazón de las humanidades. Teoría de la Educación. Revista Interuniversitaria, 34(1), 49-68. https:// doi.org/10.14201/teri. 25970

\section{RESUMEN}

Tal y como están las facultades de educación organizadas actualmente, los filósofos de la educación tienen una existencia marginal y furtiva. A medida que la educación se entiende cada vez más como una investigación científica social sobre «lo que funciona» en las escuelas, los filósofos parecen ser cada vez más inútiles. Incluso nuestro papel en la formación del profesorado, que una vez estuvo asegurado por la metáfora de los fundamentos, ahora es cuestionable. Los debates en la formación del profesorado están bastante animados: ¿los profesores necesitan más asignaturas

1. Esta es una versión revisada y aumentada de la obra The Good Life of Teaching: An Ethics of Professional Practice (Wiley-Blackwell, 2011, pp. 254-273). Me gustaría agradecer a Tania Alonso-Sainz su interés en mi trabajo, la cuidadosa atención a este manuscrito y la ayuda prestada en dirigir mis palabras hacia la atención de la audiencia de habla hispana.

Traducción: Tania Alonso-Sainz. Revisión: Ch. Higgins y Tania Alonso-Sainz. 
o más experiencia clínica? Y si necesitan más asignaturas, ¿necesitan más clases de didáctica o más conocimiento sobre la asignatura que enseñan? Nos damos cuenta así de que el tipo de experiencia que los filósofos de la educación pueden ofrecer a los profesores ni siquiera está en el marco de interés. ¿Cuál es la experiencia que los filósofos de la educación podemos ofrecer? En una palabra, es un aprendizaje liberal acerca de y para la educación. Si la educación es un espacio de preguntas humanísticas, la filosofía un amor por estas preguntas en su apertura y la filosofía educativa el oficio de mantener vivos los textos y la conversación que nos ayudan a reabrir tales preguntas, entonces la formación filosófica del profesorado es una invitación a los profesores a que sean intelectuales humanos de su campo. Es una invitación a unirse a una conversación de interés milenario. Según la concepción que he desarrollado, la Filosofía de la Educación está lejos de ser marginal. Nos ayuda a recordar cómo cada programa educativo sobre «lo que funciona» plantea preguntas clave. Y, además, recuerda a la universidad que, parafraseando el famoso ensayo de Sartre, la educación es un humanismo. Cuando la educación y las humanidades se vuelven a conectar su lugar en el centro de la universidad queda claro.

Palabras clave: Filosofía de la Educación; humanismo; universidad; formación del profesorado; Pedagogía.

\section{ABSTRACT}

As schools of education are currently organized, philosophers of education lead a marginal and furtive existence. As education comes more and more to be understood as social scientific research into "what works" in the schools, philosophers seem like a poor lot indeed. Even our role in teacher education, once secured by the metaphor of foundations, is now questionable. Debates in teacher education are lively enough: Do teachers need more coursework or more clinical experience? And if they need more coursework, do they need more classes in curriculum and instruction or more background in their "content area»? Notice, though, that the kind of experience educational philosophers are best suited to provide for teachers is not even in the picture. What is this experience? In a word, it is liberal learning about and for education. If education is a space of humanistic questions, philosophy a love of these questions in their openness, and educational philosophy the craft of keeping alive the texts and conversation that helps us re-open such questions, then philosophical teacher education is an invitation to teachers to be humane intellectuals of their field. It is an invitation to join a conversation of millennial interest. Under the conception I have advanced, educational philosophy is far from marginal. It stands to remind how each positive program of research into what works begs key questions. And it stands to remind the university that, to paraphrase the famous essay by Sartre, education is a humanism. When education and the humanities are reconnected their place at the center of the university becomes clear.

Key words: Philosophy of Education; humanism; University; teacher training; Pedagogy. 


\section{INTRODUCCIÓN}

Hay dos formas de trazar la historia de la Filosofía de la Educación en Occidente. En la primera de ellas, este campo de investigación tiene entre 50 y 100 años; en la segunda, podemos decir que ha existido durante milenios. En este artículo quiero explorar esta última historia más larga, no porque suene más noble y grandiosa, sino porque estoy convencido de que la historia del origen moderno de la disciplina nos llena de suposiciones que reducen y oscurecen lo que puede ser la Filosofía de la Educación.

La filosofía es muy anterior a su profesionalización y la Filosofía de la Educación es tan antigua como la Filosofía misma: preguntas sobre la naturaleza del aprendizaje y la enseñanza ocupan un lugar central en el trabajo de figuras fundadoras como Platón. La idea aquí no es mostrar que tenemos una larga historia de aplicación de la filosofía a la educación, sino más bien darnos cuenta de que ninguna aplicación se consideró necesaria hasta hace muy poco en la historia intelectual. Durante más de dos milenios después de Platón, las cuestiones educativas siguieron siendo fundamentales para la investigación filosófica en Occidente. Para pensadores tan diferentes como Cicerón, Agustín, Boecio, Aquino, Erasmo, Vico, Rousseau, Schiller y Oakeshott, la educación no era un tema extraño que requería reflexiones filosóficas posteriores, sino la base misma de sus investigaciones filosóficas más importantes. Hasta hace poco, nos parecía natural que los filósofos abordaran cuestiones sobre el conocimiento examinando cómo llegamos a conocer; considerando cuestiones sobre la vida buena; investigando cómo uno se vuelve virtuoso; abordando la naturaleza de la sociedad ideal a través de una discusión sobre cómo educar a los futuros ciudadanos; y contemplando la naturaleza humana preguntándose qué significa que la enseñanza y el aprendizaje sean aspectos tan fundamentales de la condición humana.

Sin embargo, cuando la filosofía se unió a las filas de las disciplinas académicas formales a finales del siglo XIX, experimentó una serie de transformaciones significativas, abandonando su tarea tradicional de articular y ejemplificar una forma de vida que pueda orientar la conducta humana, en favor de la construcción de teorías sistemáticas (Hadot, 1995). Además, la filosofía ha cedido a las ciencias sociales emergentes su preocupación tradicional por la persona en desarrollo y por prácticas sociales como la educación. Así, cuando la filosofía y la educación se unen en la disciplina moderna de la Filosofía de la Educación, lo hacen de una manera doblemente alienada. Como subcampo aplicado, se piensa que la Filosofía de la Educación está alejada de la filosofía "pura», pero además de esta se piensa que está alejada de lo práctico y lo cotidiano.

En lo que sigue, intentaré eliminar esta perversión en la lógica de nuestra autocomprensión y ofreceré lo que creo que es una forma más fructífera de comprender la filosofía, la educación y su relación, que espero resulte útil para reflexionar sobre la importancia de la Filosofía de la Educación en la investigación educativa y en la formación de los docentes. 


\section{LA FILOSOFÍA COMO AMOR A LAS PREgUNTAS ABIERTAS}

La filosofía se ha asociado con un conjunto de preguntas y de textos que plantean estas preguntas de manera explícita, pero yo diría que ambas caracterizaciones pasan por alto lo esencial y único de la filosofía. Hay algo de deshonesto en identificar retrospectivamente la filosofía con un conjunto de preguntas y sus famosos intentos de responderlas. Esto hace que parezca que las preguntas ya estaban allí. Aunque hay algo de verdad en el famoso comentario de Whitehead de que toda la filosofía occidental es una nota a pie de página de Platón, la historia de la filosofía, en ciernes, es una historia de descubrimiento de nuevas cuestiones. La filosofía, como el arte (moderno), es una actividad esencialmente rompedora. Preguntas como "What does the human subject contribute through categories and intuitions to the structuring of the world?' [¿Qué aporta el sujeto humano a través de categorías e intuiciones a la estructuración del mundo?] o 'How are power relations written into our very experience of embodiment?' [¿Cómo se escriben las relaciones de poder en nuestra propia experiencia de encarnación?] ahora nos parecen típicamente filosóficas, pero esto solo es posible porque Kant y Foucault nos enseñaron a preguntarlas. Nos provocaron a ver todo lo que había sucedido antes bajo una nueva luz, revelando preguntas que antes estaban escondidas.

Por tanto, no debemos definir la filosofía por el tipo de preguntas que se plantea. De hecho, el abanico moderno de las cuestiones filosóficas es simplemente el que no ha sido asumido por otras disciplinas. La filosofía es la disciplina que asume las cuestiones que parecen demasiado grandes, demasiado normativas o, simplemente, no empíricas para otras disciplinas. Pero también hay un sentido de la filosofía más antiguo y amplio que se recoge en el nombre de "Doctor in Philosophy» ["Doctor en Filosofía»]. Por supuesto, se puede obtener un doctorado en filosofía, pero también en botánica o lenguas y literaturas eslavas. La palabra filosofía está presente en todos los títulos de posgrado, no porque cada disertación o trabajo plantee cuestiones filosóficas en el sentido moderno, sino porque existe, o existió, una conexión esencial entre la filosofía y el aprendizaje en general. Uno no puede reclamar madurez como erudito a menos que haya dominado los métodos particulares y el canon de su campo específico, pero tampoco si no es capaz de relacionar esos detalles con el amplio espectro de conocimiento y con la situación humana. Sin esta perspectiva más amplia, el académico no obtendría una perspectiva externa de su campo; no podría reconocer si, por ejemplo, se ha obsesionado con trivialidades o se ha convertido en un formalista árido y, por lo tanto, no se podría decir que verdaderamente conozca su campo.

Como es costumbre señalar, este sentido más antiguo de la filosofía se puede ver en su etimología, a partir de philia (amor, amistad) y sophia (sabiduría). Tal vez esto, aun sabiendo los problemas de los argumentos etimológicos, podría ayudarnos a combatir la aparición de la filosofía en esta era de especialización acadmémica y profesionalización, mientras buscamos un lenguaje nuevo. "There are nowadays professors of philosophy, but not philosophers» [Hoy en día hay profesores de filosofía, pero no filósofos»], bromeó una vez Thoreau, y aunque esto se exagera para 
enfatizarlo, contiene una idea nuclear verdadera (Thoreau, 1986/1854, p. 57). Este es un buen comienzo, que interrumpe nuestra tendencia a equiparar la filosofía con la disciplina académica moderna que lleva su nombre. Pero ¿cómo podremos seguir ampliando la cuestión?

Aquí creo que el poeta Rainer Maria Rilke puede ayudarnos, ofreciendo en una de sus famosas cartas al joven Franz Kappus una elocuente descripción de la actitud filosófica:

You are so young, so before all beginning, and I want to beg you, as much as I can, dear
sir, to be patient towards all that is unsolved in your heart and to try to love the questions
themselves like locked rooms and like books that are written in a very foreign tongue.
Do not now seek the answers, which cannot be given you because you would not be
able to live them. And the point is to live everything. Live the questions now. Perhaps
you will then gradually, without noticing it, live along some distant day into the answer.
[Es usted muy joven, así que antes de empezar, quiero suplicarle, tanto como pueda,
querido señor, que tenga paciencia con todo lo que está sin resolver en su corazón
y que trate de amar las preguntas en sí mismas como habitaciones cerradas y como
libros que están escritos en una lengua muy extraña. No busque ahora las respuestas,
que no se le pueden dar porque no podría vivirlas. Y la clave es vivirlo todo. Viva las
preguntas ahora. Quizás luego, gradualmente, sin darse cuenta, vivirá un día lejano en
la respuesta] (Rilke, 1954/1901-8, pp. 34-35, cursiva en el original)

Una reacción comprensible a este pasaje (de hecho, es la que normalmente tengo) es pensar que Rilke se ha dejado llevar un poco aquí. Una cosa es advertirnos de que no debemos tratar las preguntas como un problema a resolver; pero otra es hablar de amar «the questions themselves» [las preguntas en sí mismas], lo cual sugiere una prohibición contra cualquier tipo de búsqueda, hallazgo o valoración de respuestas, porque las preguntas parecen pedir, señalar y cumplirse en las respuestas.

Hay dos peligros a evitar en relación con las preguntas. El primero, enfatizado por Rilke, es que normalmente todos somos demasiado impacientes. Molestos por la incertidumbre, tenemos dificultades para dejar que una pregunta respire antes de sofocarla con nuestra respuesta. El otro peligro, sin embargo, es que podríamos caer presos de un amor idólatra por las preguntas. Cuando hablamos de la pregunta abierta, no nos referimos, como ha demostrado Gadamer (1960/2004), a una pregunta libre de supuestos. Esto es lo que Gadamer llama una «floating question» (p. 357) ["pregunta aparente» (Gadamer, 1993, p. 225)], a diferencia de una verdadera pregunta, que no es abierta en este sentido «aparente», libre de supuestos, sino simplemente abierta. Son preguntas directas y sustantivas que tienen el poder de abrir nuevos mundos, un nuevo espacio para pensar, respirar y moverse. Si las preguntas verdaderas son dinámicas, entonces el verdadero amor por las preguntas debe implicar seguir la acción, no estancarse frente a ellas. Sin embargo, haciendo esta salvedad, podemos apreciar el desafío que nos propone Rilke de dominar nuestra impaciencia y aprender a vivir con nuestras preguntas. De esta forma, Rilke nos ofrece una nueva traducción de la filosofía: la filosofía implica intentar hacerse 
amigo de la pregunta abierta; es un amor por la apertura lo que proporciona una verdadera pregunta.

Sin embargo, incluso si aceptamos ver la filosofía bajo esta luz más amplia, podríamos preguntarnos cómo esta concepción puede aplicarse a la filosofía educativa. Después de todo, normalmente pensamos en la educación en términos de problemas y soluciones, y pensamos en la investigación educativa simplemente como el conjunto de ciencias sociales aplicadas que han surgido alrededor de una institución particular (es decir, la escuela). Una cosa es hablar de cuestionamientos abiertos en las artes liberales o incluso en las ciencias básicas, pero ¿no está fuera de lugar ese discurso aquî? Ver la educación como un espacio de preguntas requeriría un importante replanteamiento. De hecho, me exige que haga lo que los sabios siempre han sabido evitar: desarrollar y defender una definición de educación.

\section{El TRiÁngulo EdUCATIVO-FILOSÓfico: LA FORMA DE LA CONVERSACión HUMANÍSTICA}

Intentar definir la educación es notoriamente difícil. Dada la gran diversidad de objetivos e instituciones educativas, uno enfrenta un dilema. Si la definición es del todo precisa, corre el riesgo de ser tremendamente controvertida, excluyendo muchas cosas que otros consideran como las principales candidatas de la educación. Sin embargo, al intentar ser inclusivos, corremos el riesgo de una definición tan general que sea completamente vaga y poco instructiva. Por razones que se aclararán más adelante, elegiré la segunda de las vías. De hecho, para asegurarme de que mi definición sea lo más amplia posible, la construiré no en torno al sustantivo "educación", que puede tener un sesgo incorporado hacia los procesos formales y las estructuras institucionales, sino que optaré por el adjetivo 'educativo'. Y aquí está la definición que propongo: Algo es educativo si favorece el crecimiento bumano.

Como se puede ver, esta definición es intencionalmente formalista. La parte más determinante de ella, el crecimiento humano, recuerda a una de las preguntas más famosas de la historia. En la lectura de Jonathan Lear (2000, lección 1, especialmente págs. 7-25), la vaguedad del término eudaimonía de Aristóteles es una especie de seducción intencional, como si dijera: aquí está el término central alrededor del cual se construye tu vida, y, sin embargo, ni siquiera sabes lo que significa. Saber que debo perseguir mi eudaimonia es saber que debo buscar comprender mi eudaimonia: es confrontar la famosa pregunta de Sócrates, ¿cómo debo vivir? (Platón, 2004 / c. 380 a. C., 352d). Mi definición no pretende responder a la pregunta de Sócrates, sino simplemente mostrar que surge cada vez que alguien se propone recibir o dar educación. Esta definición simplemente pone de manifiesto lo que estamos haciendo cuando afirmamos que algo es educativo; estamos describiendo una relación entre cuatro términos: algo, favorece, bumano y crecimiento.

Para aventurar una afirmación educativa real, uno debe completar cada uno de estos términos con algo más concreto. Cada uno de los indicadores que he elegido es a posta ambiguo, y comienzan con «algo», que es la definición de lo indefinido. 
Este término nos recuerda que debemos preguntarnos: ¿qué (o quién) educa? Hay muchos posibles pretendientes: la soledad o la relación (¿paternidad, enseñanza, amistad o terapia?); trabajar, jugar, estudiar o viajar; naturaleza o cultura (¿obras canónicas o cultura cotidiana? ¿Creación o conservación? ¿Compromiso entre culturas o entre la misma cultura?); lenguaje, discurso o medio; instituciones o prácticas (¿juegos, artes, ciencias, humanidades u oficios?); textos, experiencias, ambientes o modelos; novedad o repetición, éxito o frustración; exploración, instrucción, observación, conversación o meditación; lo lógico o lo bello, la regla o la excepción, lo mundano o lo trascendente. El término «favorecer» también pretende ser genérico, lo que nos lleva a preguntarnos si lo educativo suele venir en forma liberadora o moldeadora; pastorear o provocar; modelar, testimoniar o dialogar; enriquecer, conservar o descartar; instruir, entrenar, iniciar o curar; ¿o quizás algo completamente diferente?

Hace un momento hablaba de "crecimiento humano" como una expresión conjunta, pero ahora me gustaría considerar estas dos palabras «humano" y "crecimiento" por separado, cada una de las cuales se erige como otro indicador sintáctico. La palabra "humano» en esta definición plantea explícitamente la pregunta de quién está siendo educado. ¿Cómo entendemos al alumno de esta propuesta educativa? ¿Qué estamos asumiendo sobre lo que es, puede hacer o necesita un ser humano? ¿Qué partes de un ser humano consideramos educables y necesitadas de educación: cuerpo o alma; corazón o mente; carácter, deseos, emociones, autocomprensión, imaginación o razón?

La palabra "crecer» nos recuerda que no se puede educar sin alguna concepción de lo que significa ser educado; no se puede decir que alguien está creciendo sin algún sentido, por tácito que sea, de qué significa esa madurez. Nuestros objetivos educativos están llenos de ideales de lo que significa una persona educada, y a su vez están incrustados en marcos normativos más amplios, en visiones del crecimiento humano. Digo visiones, en plural, porque aquí nuevamente los desacuerdos son profundos. Una persona dirá que crecer significa seguridad y prosperidad; otro contestará que significa riesgo y aventura; un tercero sostendrá que significa reducir la huella de carbono. ¿Nos realizamos mejor a través de la interioridad y la contemplación o mediante la relación y el compromiso práctico? ¿Debe medirse una vida humana por la riqueza de la experiencia, la generosidad hacia los demás, la profundidad de la percepción, la pureza de motivos, la excelencia de los logros? ¿Qué es lo más importante a considerar cuando se intenta responder a la pregunta de Sócrates: ¿placer, virtud, alegría, sabiduría, libertad, fidelidad, amplitud de miras, trascendencia, civismo, autenticidad?

Así, lejos de tratar de resolver de una vez por todas la cuestión de qué es la educación, el objetivo de definir lo educativo de esta manera es que nos planteemos las preguntas más importantes. Es una definición construida para escenificar desacuerdos, que no sugiere respuestas asentadas ni inconmensurabilidad radical. La vaguedad de los términos de mi definición invita a una diversidad de respuestas, pero al mismo tiempo reúne esas respuestas en una conversación. Es esta conversación la 
que constituye la educación en sí misma. La educación es la conversación en curso que tiene lugar en el espacio abierto por la pregunta de qué es aquello que más y mejor favorece el crecimiento humano; y consiste en las respuestas explícitas e implícitas por aquellos teóricos, prácticos y profesionales que se sienten llamados a unirse a la conversación.

Entonces, afirmar que algo es educativo supone encontrar posturas rivales y ser impulsado a un espacio de preguntas. Utilizo el plural porque claramente no es necesario plantear explícita o directamente la pregunta ‘Qué favorece el crecimiento humano?' para participar en la conversación. El objetivo de enumerar las muchas formas alternativas de tomar cada uno de los términos básicos de la definición ha sido mostrar que hay un número infinito de preguntas más pequeñas que fluyen e iluminan esta pregunta central. Sin embargo, para tener un diálogo a través de las diferencias, los participantes deben compartir lo suficiente para constituir sus diferencias como tales; de lo contrario, los interlocutores se limitan a hablar, pero nunca entre ellos. Por otro lado, si uno circunscribe demasiado desde el principio lo que hay en desacuerdo, se corre el riesgo de excluir de la conversación precisamente aquellas voces que prometen expandir el debate estrechado por supuestos no contemplados. Cada palabra de mi definición sugiere una pregunta, o un grupo de preguntas, implícitas en toda teoría y práctica educativa y concretadas en acuerdos institucionales, diseños curriculares, estrategias pedagógicas o políticas educativas. Estar involucrado en la educación significa tomar una posición en estas tres preguntas interconectadas. Es preguntarnos: cuál es nuestra naturaleza; qué constituye el crecimiento humano individual y colectivo; y qué nos mueve hacia ese bien dada nuestra condición. Esta pregunta compuesta nos ayuda a articular los amplios márgenes de la investigación educativa, como se ilustra en la Figura 1 que llamo El triángulo educativo-filosófico.

Según el modelo que he propuesto, hay tres tipos de cuestiones que a menudo se consideran distintas - la antropológico-filosófica, la ético-política y la pedagógica- que están íntimamente relacionadas, y constituyen aspectos de la cuestión educativa básica.

A esto, los escépticos pueden responder que, si bien está muy bien pretender que los educadores se interesen por las preguntas humanistas de la parte inferior del triángulo, llamar a las tres categorías educativas no parece correcto. Mi respuesta es que, al vincular una pregunta obviamente educativa con dos tradicionalmente humanistas, estoy recordando simultáneamente a las humanidades sus raíces educativas y recordando a la educación sus dimensiones humanistas. Para corroborar esto, consideremos más a fondo la naturaleza de cada pregunta, por qué cada una es ineludible para los educadores y cómo las tres están interconectadas.

Primero, consideremos el vértice ético del triángulo. Sin una visión de qué es la vida buena para los seres humanos, los educadores no podrían tomar las innumerables decisiones educativas cualitativas que, de hecho, toman. Cuando los profesores deciden adoptar este tono en lugar de aquel, o incluir una actividad en lugar de otra, lo hacen porque piensan que será mejor para sus alumnos. Pero 
"mejor» es solo una forma de decir "más cerca de lo bueno", y sobre cuestiones de bien no hay respuestas fáciles. Por lo tanto, debajo de las opciones educativas, incluso aparentemente superficiales, se encuentran profundas cuestiones normativas. Sin una idea de en qué deberíamos estar evolucionando, ¿̨cómo podríamos decir si un cambio es para mejor? Cuando nos esforzamos por ayudar a alguien a madurar, confiamos en una visión de lo que es la madurez. Cuando instruimos, confiamos en una visión de lo que es importante aprender y, por lo tanto, en lo que constituye una persona verdaderamente educada. Estas cuestiones nos llevan a su vez a las preguntas fundamentales de la ética: ¿Qué hace que la vida sea significativa o rica? ¿Cuáles son las virtudes humanas más importantes? ¿Qué es el bien colectivo? ¿Cómo deberíamos vivir mejor juntos? Los educadores, se planteen profundamente o no estas preguntas, al menos deben haberlas eliminado con alguna respuesta rápida, ya que nadie puede intentar fomentar el crecimiento humano sin una visión del crecimiento humano, individual o colectivo.

FIGURA 1

EL TRIÁNGULO EDUCATIVO-FILOSÓFICO

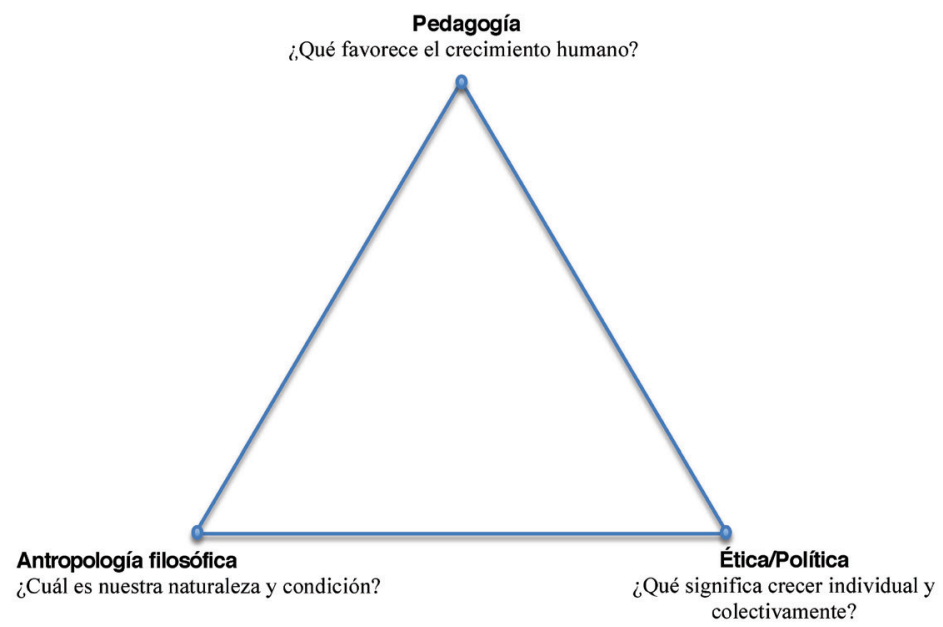

Fuente: Elaboración propia

No podemos comprender verdaderamente el crecimiento humano de antemano, o abstraídos del deseo de convertirnos en algo bueno o de nuestros esfuerzos por fomentar el desarrollo humano. Así como nuestras visiones del crecimiento humano llenan de contenido a nuestras pedagogías, nuestro conocimiento de lo que nos acerca a lo bueno sirve para enseñarnos algo sobre el bien mismo. 
Es por esta razón que he etiquetado el vértice superior del triángulo como "pedagogía» en lugar de "educación»: las preguntas sobre lo que favorece el crecimiento son solo un aspecto de la conversación educativa más amplia. Como señalé anteriormente, aquí hay dos tipos de preguntas estrechamente relacionadas: preguntas sobre qué podría constituir el catalizador principal del desarrollo (relaciones, entornos, textos, etc.) y preguntas sobre qué metáfora muestra mejor esta catálisis (nutrir, instruir, desafiar, etc.).

Aquí debemos agregar una tercera coordenada a este mapa de cuestiones educativas. Utilicé la palabra "humano" para referirme, sin resolverlos, a los debates sobre quién o qué se está educando, sobre la naturaleza, las necesidades y las capacidades de nuestros futuros estudiantes. Debajo de las diferencias en los debates radica el hecho de que cada acción y documento educativo está cargado de suposiciones sobre quiénes somos y por qué necesitamos educación, sobre qué partes de los seres humanos son educables y cuáles no. Teorizar o practicar la educación es unirse a la larga conversación que llamo antropología filosófica, planteando preguntas como: ¿Qué nos motiva? ¿Cuáles son nuestras capacidades, necesidades y debilidades fundamentales? ¿Qué es la condición humana? ¿Qué es la naturaleza humana? ¿Somos seres esencialmente racionales, apetitivos o imaginativos, o somos de naturaleza tan esencialmente cultural o histórica que no se justifican tales generalizaciones a lo largo del tiempo y el lugar? Esto no sugiere, por supuesto, que los educadores piensen o deban pensar en estas cuestiones en términos tan abstractos y grandiosos. Los educadores están haciendo antropología filosófica cuando hablan de 'niños', 'carácter' o 'necesidades emocionales', o cuando justifican sus acciones con referencia a lo que un niño puede hacer, cómo de debe enseñar a los niños con dificultades, cómo los niños y las niñas aprenden de manera diferente, lo que motiva a un estudiante enfadado, etc.

Enseguida veremos de cerca la relación entre los dos vértices inferiores del triángulo, pero antes me gustaría hacer una observación. Aunque todas las teorías educativas tienen tanto una antropología como una visión de florecimiento, una u otra puede quedar implícitas. Por ejemplo, la psicología del desarrollo a menudo se introduce en los debates educativos como si los datos sobre cómo nos desarrollamos pudieran resolver por sí solos la cuestión de cómo debemos educar. Digamos que aquello en lo que deberíamos desarrollarnos queda más o menos implícito. En otras ocasiones, es el ideal ético o político el que se pone en primer plano con supuestos clave sobre nuestra naturaleza y condición que operan en segundo plano. Detrás de los llamamientos a educar a los ciudadanos, los virtuosos o los pensadores críticos se esconden suposiciones sobre los «salvajes", el "talento en bruto» o la "falsa conciencia». Podríamos decir del educador lo que diríamos del escultor: que las herramientas elegidas y las formas que intentan dar son diferentes si se trabaja en mármol o arcilla. O podríamos rechazar esta metáfora pedagógica de moldear y modelar como inherentemente mala educación. Pero obsérvese que nuestra crítica entonces se basaría en otra visión de la condición humana, sosteniendo que los seres humanos son esencialmente libres, dignos, guiados por un daimon interno 
o sujetos a alguna lógica natural de desarrollo. No se puede educar sin tomar una posición en la subconversación que he estado llamando antropología filosófica.

\section{TAYLOR Y EL ARGUMENTO DE LA INTERDEPENDENCIA FUERTE}

Acabamos de ver algunos ejemplos de cómo las concepciones de la naturaleza y el crecimiento humanos se entrelazan en nuestro pensamiento educativo, pero consideremos el asunto más de cerca. Después de todo, la filosofía contemporánea se divide en subcampos: algunos filósofos se dedican a la teoría política, otros a la filosofía de la mente y otros se especializan en ética. En la Parte I de Sources of the Self, Charles Taylor desafía la sostenibilidad de esta división del trabajo filosófico, desarrollando argumentos convincentes para la interdependencia de puntos de vista éticos y filosófico-antropológicos. El primer argumento de Taylor comienza con la observación de que «most urgent and powerful cluster of demands we recognize as moral» [el conjunto de demandas más urgentes y poderosas que reconocemos como morales] implican el respeto por la vida y la integridad de otras personas (Taylor, 1989, p. 4). Estas demandas a menudo se experimentan en un "gut level» [nivel instintivo], pero siempre implican, según Taylor, «acknowledgments of claims concerning their objects» [reconocimientos de afirmaciones/valoraciones referidas a los objetos] (Taylor, 1989, p. 7).

Esto significa que al hacer juicios morales o al sentir emociones morales, confiamos en una variedad de nociones ricas y complejas (aunque rara vez articuladas) sobre cómo son las cosas y por qué son importantes y valiosos los seres humanos; y estos puntos de vista integrados son de naturaleza "ontológica».

Sin embargo, Taylor no se para aquí, al darse cuenta de que plantea una cuestión importante. Si los juicios morales involucran ideales sustantivos, que se basan en creencias sustantivas de fondo sobre cómo son las cosas y las personas, entonces parte de este fondo ontológico concierne a la antropología filosófica. Pero Taylor reconoce que muchos niegan que los juicios morales requieran este tipo de trabajo filosófico. En una concepción comúnmente extendida (que podría ser denominada naturalismo filosófico aliado al subjetivismo moral), la moralidad se ve, bien como un conjunto de tabúes interiores para ayudar a que la sociedad funcione sin problemas, o como la proyección de preferencias subjetivas en el mundo. Para los defensores de este punto de vista, los relatos ontológicos de la naturaleza humana y del crecimiento son más que irrelevantes para la ética, son cuentos de hadas metafísicos premodernos de los que las personas racionales deben aprender a prescindir.

A la luz de esta objeción, Taylor lanza un segundo argumento, pasando de una pregunta externa sobre la obligación moral (¿cómo se debe tratar a otros seres humanos?) a una perspectiva ética interna. Taylor piensa que la mayoría de las evaluaciones sólidas que hacemos en nuestras vidas se extienden más allá de la moralidad propiamente dicha. Cuando nos preguntamos si nuestras vidas son significativas o ricas, cuando nos preocupamos por nuestra dignidad, felicidad o virtud, o luchamos por la forma que deberíamos dar a nuestras vidas, estamos 
comprometidos con una reflexión ética. Por lo tanto, Taylor trabaja contra el «naturalist temper» [temperamento naturalista] moderno que etiquetaría consideraciones éticas primordiales como mera preferencia estética o estupidez espiritual (p. 19). Taylor llama a este segundo argumento "fenomenológico" porque procede de elementos comunes de la experiencia sentida.

La observación fenomenológica significa que la gente a menudo se hace la desconcertante pregunta "¿quién soy yo?». Es desconcertante porque uno pensaría que no tendría que preguntársela o que solo necesitaría recordar su "name and genealogy" [nombre y genealogía] (Taylor, 1989, p. 27) para responderla. Y esto le lleva a la siguiente deducción: "the condition of there being an identity crisis is precisely that our identities define the space of qualitative distinctions in which we live and choose» [la condición de que haya una crisis de identidad es precisamente que nuestras identidades definan el espacio de distinciones cualitativas en el que vivimos y elegimos] (Taylor, 1989, p. 30). Es decir, que la identidad no sería una categoría tan destacada para nosotros si no fuera por las distinciones éticas que encarna esta noción, esto es, si la identidad no fuera algo a lo que uno podría ser fiel o traicionar. Conocer el nombre y el número identidad de uno, no responde a la cuestión de la identidad, que no solo pregunta quién eres, sino dónde te encuentras en relación con lo que te importa y si te ha dedicado, entonces, a esas cosas importantes. Por lo tanto, el proceso de comprendernos a nosotros mismos (antropología filosófica) no puede llevarse a cabo sin el uso de distinciones evaluativas fuertes (ética). Como dice Taylor:

What this brings is the essential link between identity and a kind of orientation. To know who you are is to be oriented in moral space, a space in which questions arise about what is good or bad, what is worth doing and what not, what has meaning and importance for you and what is trivial or secondary (1989, p. 28). [Lo que esto explica es el vínculo esencial entre identidad y una especie de orientación. Saber quién eres es estar orientado en el espacio moral, un espacio en el que surgen preguntas sobre qué es bueno o malo, qué vale la pena hacer y qué no, qué tiene significado e importancia para ti y qué es trivial o secundario.]

El objetivo del argumento es mostrar la irrealidad del escepticismo naturalista sobre si existen marcos referenciales tan densos. La vida humana ya está llena de dudas, marcada por secuencias de orientación, desorientación y reorientación. Sin embargo, esa duda sobre uno mismo solo tiene sentido en el contexto del trasfondo de suposiciones de lo que importa. Por lo tanto, dudar de la existencia de los marcos en sí tiene poco sentido.

Taylor profundiza en este vínculo entre identidad y evaluación fuerte a través de una segunda observación fenomenológica relacionada: nos damos sentido a nosotros mismos a través de historias, "we grasp our lives as a narrative» (Taylor, 1989, p. 47) [captamos nuestras vidas como una narrative]. "What I am» [Lo que soy], escribe Taylor, «has to be understood as what I have become» [tiene que entenderse 
como aquello que he llegado a ser] y en relación con "what I project to become» [lo que proyecto llegar a ser] (Taylor, 1989, p. 47).

Cuando nos hacemos la pregunta "iqué he llegado a ser?», no lo hacemos con la fría neutralidad del científico de laboratorio, sino que nos referimos a «¿dónde me encuentro en relación con lo que considero bueno?». Tales historias son siempre historias éticas, aunque no siempre explícitamente. La dimensión normativa puede aparecer en forma de adverbio humilde o adjetivo vago. Sin embargo, cuando informamos que estamos "haciéndolo bien" o "nos sentimos estancados", los ideales éticos acechan en el trasfondo.

Aquí necesitamos recordar la distinción de Taylor entre las nociones de evaluación débil y evaluación fuerte. Según la teoría débil, consideramos las cosas buenas porque las preferimos; según la teoría fuerte, preferimos las cosas porque las consideramos buenas. "Orientation to the good" [La orientación hacia lo bueno], escribe Taylor, «is not an optional extra» [no es un extra opcional] para dar sentido a nuestras vidas y no existe una vida humana que "makes absolutely no sense to the person who lives it» [no tenga absolutamente ningún sentido para la persona que la vive]. 'What is basic, then, to every human life as it is lived' [Lo que es básico, entonces, para toda vida humana tal como se vive], concluye Taylor, «is the operation of, more or less explicit, substantive ethical categories» [es el funcionamiento de categorías éticas sustantivas, más o menos explícitas]. Para Taylor, entonces, hay dos preguntas estrechamente relacionadas que necesariamente surgen para todas las personas: «QQuién soy yo?» y "¿Dónde me encuentro en relación con lo que entiendo que es bueno?». En este sentido, todos somos «ontólogos morales» en nuestras vidas cotidianas.

Con la ayuda de Taylor, ahora podemos ver la conexión inseparable de dos de las tres preguntas que definen el triángulo filosófico-educativo. Pero como ya he indicado, lo pedagógico sirve como una bisagra obvia entre estas otras dos cuestiones básicas. Dada nuestra naturaleza y lo que es bueno para nosotros, la pregunta pedagógica es: ¿qué es lo que mejor favorece nuestro crecimiento? En el relato de Taylor, esta bisagra pedagógica entre cuestiones antropológicas y éticas se deja en gran parte implícita, pero no es difícil de localizar en cada uno de sus estudios de caso. Taylor comienza con Platón, enfatizando la interconexión entre la ética de Platón del autodominio racional y su concepción del alma dividida en partes superiores e inferiores, y capaz de sintonizarse con el orden del cosmos. Aunque Taylor no lo resalta tanto como podría, sí observa cómo la ontología moral de Platón se basa en una intuición pedagógica crucial, la distinción entre instrucción y conversión. En el centro de la famosa 'Alegoría de la Caverna' y de la República en su conjunto está la idea de que la forma más elevada de educación no es la de impartir habilidades o conocimientos, sino la de 'dirigir el alma' hacia objetos dignos de nuestra atención (Platón, 2004 / c. 380 a. C., página 210 [Libro 217, 516b-d]). Y esto ni siquiera tiene en cuenta la pedagogía dialógica promulgada en los diálogos de Platón. Si uno lee a Platón por lo que muestra tanto como por lo que dice, entonces un diálogo como el Menón es principalmente sobre pedagogía y solo secundariamente sobre 
la psiqué (específicamente, su capacidad de recordar) y lo bueno (específicamente, excelencia del carácter). (Platón, 1961 / c. 380 a. C.).

O consideremos las discusiones de Taylor sobre Descartes y Montaigne. Lo que Taylor quiere que veamos sobre Descartes es la forma en que anuncia el paso de la racionalidad como una cualidad del universo al que nos alineamos con un procedimiento interno que utilizamos. Pero resulta que el famoso "método de regular correctamente la razón" de Descartes es una pedagogía autodidacta, un proceso meditativo y escéptico mediante el cual uno expulsa creencias defectuosas y construye una base más firme para la creencia verdadera. Descartes introduce explícitamente sus famosos experimentos mentales como el siguiente paso en su intento continuo de asegurar una educación sólida después de haber probado tanto la escolarización formal como los viajes (Descartes, 1988/1637, Parte 1). En el centro del proyecto de Montaigne está un tipo diferente de proceso de autoeducación. Como muestra Taylor, comprender la ética de Montaigne de la autoconciencia y la autoaceptación y su visión del yo como inherentemente inestable va de la mano de la comprensión de su proyecto novedoso, su crónica de impresiones personales y su autoestima (Taylor, 1989, pp. 178-181).

\section{OBJECIONES E IMPLICACIONES}

De esta manera, Taylor nos ayuda a percibir las estrechas conexiones entre estas tres categorías de preguntas y a profundizar en el sentido que le damos tanto a la centralidad de la educación en la historia de las ideas como a la importancia de las perspectivas humanistas en la educación. Estas tres preguntas: ¿QQuiénes somos?», "¿En qué deberíamos convertirnos?», "¿Qué nos mejora?» son las preguntas humanistas por excelencia. Están iluminadas por todas las obras humanísticas, excepto las más estériles, e incluyen no solo textos de una variedad de disciplinas modernas, sino también obras religiosas, belles letres, y artes. El estudio del bien no se limita a la filosofía, la política y la religión; ni los ministerios de educación tienen el monopolio de la cuestión de la transformación humana. Y por supuesto, todas las humanidades, como su propio nombre sugieren, son intentos de iluminar lo que significa ser humano mediante el estudio de nuestra vida interior y nuestros artefactos (lenguajes y leyes, obras de arte y edificios, teorías y religiones, tratados y costumbres sexuales).

La implicación de esto, como he sugerido, es doble: ser un educador reflexivo es convertirse en un estudiante de humanidades; pero también, ser un verdadero estudioso de las humanidades, ser un intelectual humano, es preocuparse por las cuestiones educativas. Lo que revela el triángulo es que, lejos de ser una ciencia social aplicada de reciente invención que acompaña a la institución escolar, la educación es el principio organizador de las bumanidades. La educación es lo que pone en relación e ilumina las cuestiones humanísticas básicas. El triángulo también rechaza los aspectos de la autoimagen actual de las humanidades. La concepción de las humanidades como disciplinas separadas tiende a desalentar el tipo de 
pensamiento sinóptico que ilumina las dimensiones e interconexiones educativas entre las humanidades; y la idea del trabajo humanista como investigación tiende a desalentar el reconocimiento de que las humanidades no son meramente métodos de conocimiento, sino modos de autoconocimiento que perseguimos, no desinteresadamente, sino con la mirada puesta en nuestro propio crecimiento individual y colectivo.

Aquí queda claro un problema. He estado argumentando que la educación es una conversación en curso sobre el devenir humano provocada por preguntas características y sostenida por la disposición (filosófica) de valorar estas preguntas en su propia apertura. El problema surge cuando recordamos una de las conclusiones de nuestra discusión sobre Taylor, a saber, que el estudio de las cuestiones del devenir humano es inevitablemente de naturaleza histórica. Las creencias densas, evaluativas, discutibles, más o menos explícitas sobre la naturaleza y el crecimiento humanos que él llama ontología moral encuentran su lugar dentro de los lenguajes que se sitúan a su vez en las tradiciones de pensamiento y en los horizontes de cada época. La forma de las preguntas disponibles en un momento dado o dentro de cualquier tradición particular de pensamiento siempre representan una interpretación de las preguntas, o una respuesta parcial a ellas. Un signo de interrogación no garantiza una verdadera pregunta abierta. Uno de los requisitos de la verdadera pregunta que concluimos fue que una pregunta necesita un interrogador; tiene que ser planteado por una persona de carne y hueso que siente un conflicto sobre algún tema de importancia para él o ella. Las preguntas no permanecen abiertas porque alguien (como yo) las enumere en una lista. La pregunta abierta es, de hecho, una excepción. Estamos inundados de obviedades, creencias tan fundamentales para nuestra forma de estar en el mundo que no nos parecen creencias. Cuando nos encontramos en las garras de una pregunta real, lo que hacemos es poner en primer plano algunos aspectos del mundo, pero por definición dejamos muchos otros en segundo plano. Formar parte de una manera de hablar, de una tradición de pensamiento ligada a un tiempo y un lugar, significa que determinadas cuestiones nos cautivan más que otras, y estar abiertos en una dirección tiene el precio de una ceguera radical en otra.

Esto plantea dos dificultades relacionadas para el triángulo. Primero, ¿cómo puedo afirmar que estoy haciéndome preguntas abiertas cuando solo aparecen en un momento y en un lugar restringido y excluido, y yo mismo estoy situado en una posición tan contingente? En segundo lugar, al definir la educación en torno a cuestiones tan vagas como qué es la condición humana, ¿no estoy ofreciendo a los educadores ejemplos perfectos de esa especie de pseudopregunta que, como señalamos antes, Gadamer llama la pregunta flotante?

Permítanme comenzar mi respuesta reconociendo mi situación. Como todo el mundo, mi enfoque de las cosas depende de aspectos de mi historia y mi entorno, muchos de los cuales operan completamente "behind my back" [a mis espaldas], como diría Gadamer (Gadamer, 1976/1967, p. 38). Sin embargo, que no tenga una visión de lo que hay «behind my back» no significa que no se pueda avanzar en la 
ampliación de los horizontes. Dedicándonos a desenterrar presupuestos ocultos y viendo más allá de reducciones falsas, podemos avanzar en la eliminación de parte de la miopía que conlleva nuestra situación. En otras palabras, aunque mi relato ciertamente no presume omnisciencia, prescribe el proyecto de intentar liberarse del provincialismo tanto como sea posible.

Aún así, la cuestión sigue siendo cómo puedo tener la clara pretensión de afirmar que estas tres preguntas educativas son abiertas y fundamentales. ¿Estoy sugiriendo que todos los demás están luchando lentamente hacia una mentalidad abierta mientras yo mismo ya estoy allí, escribiendo para recomendar a todos mi postura? Claramente no: el triángulo está pensado como un dispositivo heurístico. Y obsérvese cómo, al introducir sus tres vértices, me encontré recurriendo a dos estrategias. Por un lado, etiqueté cada esquina con indicadores extremadamente amplios y vagos (¿Qué favorece el crecimiento? ¿Cuál es la condición humana?). Por otro lado, elaboré listas de cuestiones rivales características de cada vértice. Según mi definición, la educación es un espacio de desacuerdo, pero, por supuesto, los desacuerdos rara vez tratan solo de respuestas. Los desacuerdos más importantes son los desacuerdos sobre las preguntas. El debate sobre el aborto sería mucho más sencillo si se tratara de un debate entre pro-vida y anti-vida o entre defensores del derecho a decidir y defensores de la no-elección.

Tomemos el ejemplo de la antropología filosófica. No se puede formular una pregunta única que sea lo suficientemente amplia como para definir todo el campo de cuestiones de una vez por todas. Históricamente, la pregunta filosófico-antropológica por excelencia era "¿Qué es el hombre?», una pregunta que ahora nos parece peligrosamente parroquial y universalizadora a la vez. Fue bueno dialogar sobre ideas, pero lo hizo a costa de sumergir otras. Ahora podemos ver cómo asumir que uno podría usar el pronombre masculino como un universal de género neutro hizo invisibles áreas enteras de investigación (relacionadas con el papel del género en la experiencia humana), que son ahora centrales en nuestros esfuerzos por descubrir qué nos hace humanos.

Esto nos demuestra que hacer la pregunta de una manera determinada es meternos de lleno en la controversia. Por lo tanto, ninguna pregunta puede presentarse como la cuestión filosófico-antropológica fundamental o general, porque cada pregunta ya presupone una serie de supuestos filosófico-antropológicos, iluminando una parte del campo y oscureciendo otra. No debemos buscar una sola pregunta lo suficientemente neutral como para abrir todas las preguntas posibles sobre los seres humanos de una vez por todas. Esto realmente equivaldría a una pregunta flotante. Una pregunta que sea general no abriría puertas; estaría vacía, sería de poca o ninguna importancia. Se abren nuevas perspectivas sobre lo humano captando los límites de las antiguas, preguntando qué omite, oscurece o distorsiona una determinada visión. El campo en su conjunto, entonces, está mejor representado por un conjunto de preguntas relacionadas que, en su proximidad, resaltan los truncamientos en cada una, al tiempo que apuntan al terreno común que debe existir para que estas 
incluso constituyan supuestos rivales. El terreno común se puede intuir, pero, en última instancia, no nombrar.

Ni siquiera una larga lista de preguntas podría mapear colectivamente los límites completos de la conversación, ya que una pregunta genuinamente nueva no se une como una más entre la multitud, sino que empuja todo a un lado en un cambio de perspectiva. Aprender a hacer una nueva pregunta requiere más que poner un signo de interrogación al final de alguna oración que aún permanece intelectualmente declarativa para nosotros. Uno debe ser capaz de considerar seriamente una alternativa para cuestionar algo.

Por lo tanto, el triángulo completamente abierto es una ficción, y las preguntas que describo son solo hipotéticamente abiertas. Las nombro con indicadores banales y formalistas, con la finalidad de marcar puntos para posibles expansiones y revisiones. He elegido indicadores deliberadamente vacíos, incluso incómodos (por ejemplo, "favorece», "crecimiento» y "ético-político») para apuntar hacia una apertura que solo podemos inferir. Pero, aunque sea ficción, es una ficción útil. Su apertura formalista nos invita a poner puntos de vista concretos y rivales de las tradiciones en una distancia crítica entre sí dentro de un diálogo que permite que se produzca una fusión de horizontes y que podamos vislumbrar una cuestión sustantiva más abierta. En cualquier momento y lugar, nos encontraremos limitados por el horizonte de nuestras suposiciones. Y, por supuesto, encontramos aún más reducciones dentro de los horizontes de nuestra época.

Consideremos, en primer lugar, el supuesto común de que educación significa educación formal e intencional, y añadamos que la educación formal son las escuelas públicas contemporáneas. Muchas vías de investigación educativa son excluidas tan pronto como consideramos al alumno como un estudiante de instrucción obligatoria y formal y aceptamos toda la arquitectura de las aulas, cursos, asignaturas, calificaciones y exámenes. Y, por supuesto, en la práctica, las reducciones van mucho más allá. Veamos brevemente cada vértice del triángulo y sus reducciones típicas.

La conversación completa sobre el crecimiento individual y colectivo está casi siempre representada por una consideración reduccionista de nuestros ideales de la persona educada. Pero, por supuesto, es igual de raro mantener una discusión amplia sobre los objetivos educativos. De hecho, en una esquina del triángulo hay un puñado de objetivos curriculares. Que esto representa una dieta escandalosamente pobre del régimen completo de cuestiones éticas y políticas puede demostrarse de dos formas, una formal y otra sustantiva. La queja formal es que sustituimos un ritual nominalista y burocrático (cada lección debe tener un objetivo claro que debe ser escrito brevemente en la programación de aula y, según la escuela, también en la pizarra para que los estudiantes lo vean) por una búsqueda genuina, interesante y de búsqueda sobre lo que significa ser educado y prosperar individual y colectivamente. La queja sustantiva es que no sólo nuestros objetivos educativos se reducen a etiquetas y clichés, sino que la escolarización moderna y obligatoria se organiza en torno a una visión muy pobre de lo que significa la persona educada. El carácter, la emoción y la imaginación nunca han sido el punto fuerte de la escolarización, 
en la que la tendencia ha sido reducir el aprendizaje a la memorización de información y al dominio de habilidades. En esta nueva era de la fiebre por medir, el empobrecimiento va aún más lejos a medida que se gasta más y más energía en la escuela para ayudar a los estudiantes a mejorar sus calificaciones en las pruebas estandarizadas de matemáticas y lectura.

Pasando de la ética a la antropología filosófica, encontramos una reducción similar a la evidenciada en el plan de estudios de formación docente. La vasta y variada conversación sobre lo que significa ser humano se reduce a un curso de psicología educativa, una unidad sobre estilos de aprendizaje y unas lecturas sobre multiculturalismo. Conceptos como etapas de desarrollo y tipos de personalidad están siempre en nuestras conversaciones, lo que nos permite hacer ciertas afirmaciones sobre de qué están hechos los seres humanos, mientras que al mismo tiempo nos impiden enunciar muchas preguntas filosófico-antropológicas más amplias.

FIGURA 2

HORIZONTES DE LA PRÁCTICA EDUCATIVA

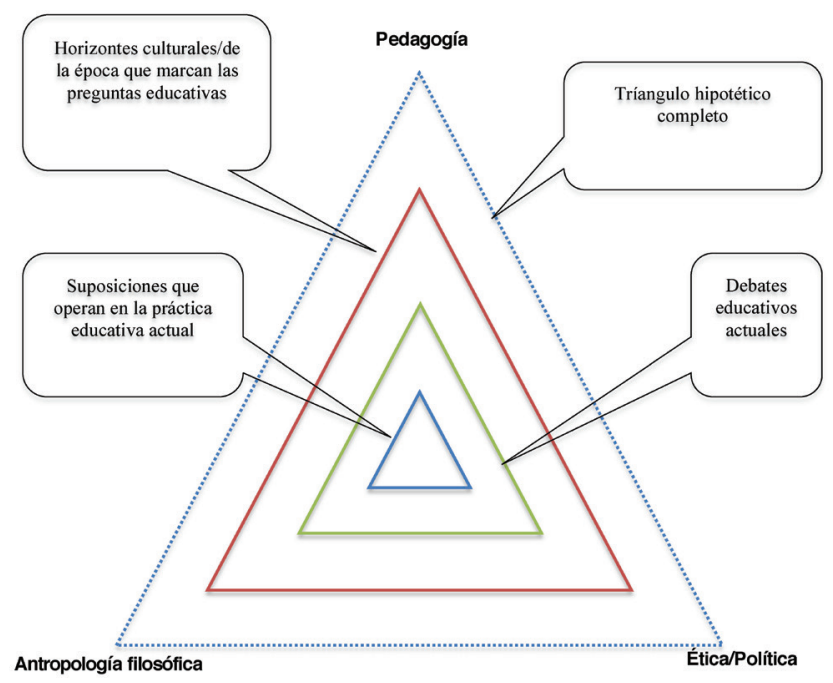

Fuente: Elaboración propia

Incluso en la esquina pedagógica del triángulo encontramos una reducción significativa de la cuestión. Las técnicas de gestión del aula y otros métodos de instrucción tienden a representar una consideración más amplia de lo que favorece el crecimiento humano. En todas estas áreas, vemos una tendencia a quedar atrapados en un debate que oculta la exclusión de toda una gama de consideraciones clave. Por lo tanto, quedamos hipnotizados entre una y otra teoría de la etapa de 
desarrollo, y no nos damos cuenta de que las preguntas previas y cruciales nos están "gritando" (por ejemplo, las preguntas sobre el lugar de la psicología del desarrollo moderna en la conversación filosófica-antropológica más amplia). Y, por supuesto, un cliché es demasiado indeterminado para poder enseñar, porque el maestro necesita respuestas plausibles a la mayoría de las preguntas educativas que tiene. La Figura 2 evoca los horizontes anidados de la práctica educativa.

\section{ConCLUSIón}

Tal y como están las facultades de educación organizadas actualmente, los filósofos de la educación tienen una existencia marginal y furtiva. A medida que la educación se entiende cada vez más como una investigación científica social sobre "lo que funciona" en las escuelas, los filósofos parecen ser cada vez más inútiles. Leemos despacio (¡leemos!), frecuentemente cometemos el pecado capital de citar obras de más de 10 años de antigüedad, y además no tenemos datos. Incluso nuestro papel en la formación del profesorado, que una vez estuvo asegurado por la metáfora de los fundamentos, ahora es cuestionable. Los debates en la formación del profesorado están bastante animados: ¿̇los profesores necesitan más asignaturas o más experiencia clínica? Y si necesitan más asignaturas, ¿necesitan más clases de didáctica o más conocimiento sobre la asignatura que enseñan? Nos damos cuenta así de que el tipo de experiencia que los filósofos de la educación pueden ofrecer a los profesores ni siquiera está en el marco de interés.

¿Cuál es esta experiencia que los filósofos de la educación podemos ofrecer? En una palabra, es un aprendizaje liberal acerca de y para la educación. Esto no es lo mismo que la formación práctica en el oficio de enseñar o que las bases de las ciencias de la educación (si es que tales ciencias debieran madurar alguna vez). Tampoco debe confundirse con el dominio de un arte liberal como área de contenido (que en cualquier caso roza con una contradicción los términos). Si la educación es un espacio de preguntas humanísticas, la filosofía un amor por estas preguntas en su apertura y la filosofía educativa el oficio de mantener vivos los textos y la conversación que nos ayudan a reabrir tales preguntas, entonces la formación filosófica del profesorado es una invitación a los profesores a que sean intelectuales humanos de su campo. Es una invitación a unirse a una conversación de interés milenario.

Según la concepción que he desarrollado, la Filosofía de la Educación está lejos de ser marginal. Nos ayuda a recordar cómo cada programa educativo sobre "lo que funciona" plantea preguntas clave. Y, además, recuerda a la universidad que, parafraseando el famoso ensayo de Sartre, la educación es un humanismo. Cuando la educación y las humanidades se vuelven a conectar, como se ilustra en la figura triangular que he ofrecido aquí, su lugar en el centro de la universidad queda claro. 


\section{REFERENCIAS BIBLIOGRÁFICAS}

Arrowsmith, W. (1966). The Shame of the Graduate Schools. Harper's Magazine, 232(1390), 547-558.

Arrowsmith, W. (1967a). Graduate Study and Emulation. College English, 28(8), 547-558.

Arrowsmith, W. (1967b). The Future of Teaching: The Molding of Men. The Journal of Higher Education, 38(3), 131-143.

Arrowsmith, W. (1971). Teaching and the Liberal Arts: Notes Toward an Old Frontier. En D. Bigelow (Ed.), The Liberal Arts and Teacher Education: A Confrontation (pp. 5-15). University of Nebraska Press.

Barrow, R. (1983). Does the Question «What is Education?» Make Sense? Educational Theory, 33(3-4), 191-196.

Descartes, R. (1988). Discourse on the Method. Cambridge University Press.

Dewey, J. (1916). Democracy and Education: An Introduction to the Philosophy of Education. The Macmillan company.

Gadamer, H.-G. (1976/1967). On the Scope and Function of Hermeneutical Reflection. En D. E. Linge (Ed.), Philosophical Hermeneutics (pp. 18-43). University of California Press.

Gadamer, H.-G. (1993). Verdad y método. Ediciones Sígueme.

Gadamer, H.-G. (2004). Truth and Method. Continuum. 2nd rev.

Higgins, C., Mackler, S., \& Kramarsky, D. (2005). Philosophy of Education. En S. J. Farenga, \& D. Ness (Eds.), Encyclopedia of Education and Human Development (Vol. I, pp. 215-236). M.E. Sharpe.

Johnson, T. (1995). Discipleship or Pilgrimage? The Educator's Quest for Philosophy. SUNY Press.

Langer, S. K. (1953). Feeling and Form: A Theory of Art, Developed from Philosophy in a New Key. Charles Scribner's Sons.

Lear, J. (2000). Happiness, Death, and the Remainder of Life: The Tanner Lectures on Human Values. Harvard University Press.

Nietzsche, F. (1990/1874). Schopenhauer As Educator. En W. Arrowsmith (Ed.), Unmodern Observations: Unzeitgemässe Betrachtungen (pp. 147-226). Yale University Press.

Nietzsche, F. (1990/1874-5). We Classicists. En W. Arrowsmith (Ed.), Unmodern Observations: Unzeitgemässe Betrachtungen (pp. 305-388). Yale University Press.

Plato. (1961/c. 380 B.C.E.). Meno. En E. Hamilton, \& H. Cairns (Eds.), The Collected Dialogues of Plato (pp. 353-384). Princeton University Press.

Plato. (2004). Republic. Hackett.

Rilke, R. M. (1954). Letters to a Young Poet (Rev.). W.W. Norton \& Co.

Rorty, R. (1989). Contingency, Irony, and Solidarity. Cambridge University Press.

Taylor, C. (1989). The Sources of the Self: The Making of the Modern Identity. Harvard University Press.

Thoreau, H. D. (1986/1854). Walden. En Walden and Civil Disobedience (pp. 43-382). Penguin. 